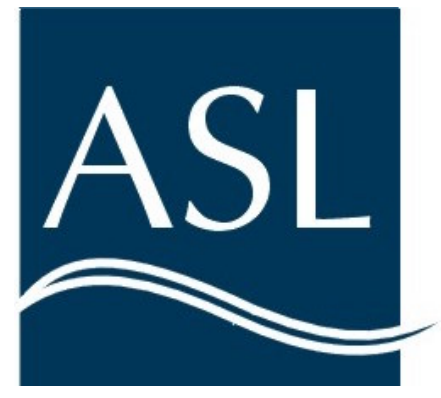

\title{
A DIGITAL PROGRAMMABLE HIGH RESOLUTION 200KHZ WATER COLUMN PROFILER
}

\author{
Ward W. Cartier and David D. Lemon \\ ASL Environmental Sciences Inc. \\ 1986 Mills Rd., Sidney, B.C. V8L 5 Y3 Canada
}

Proceedings from Oceans '99 in Seattle, Washington, September 1999.

\begin{abstract}
Acoustical instrumentation for imaging the water column has traditionally been ship mounted and configured to work in a downward looking orientation. Until recently, data acquisition for these acoustic instruments has used an analog strip chart recorder tended by an operator on board a surface vessel. With the advent of digital electronics, digital echo sounders have begun to appear. However, these instruments have followed the traditional configuration and, for the most part, have remained shipboard mounted. The past five years has seen unprecedented growth in the development of low power digital microprocessor, storage and data acquisition technologies.
\end{abstract}

An acoustical $200 \mathrm{kHz}$ high resolution, digital, programmable Water Column Profiler (WCP) has been developed incorporating these new technologies. The WCP offers unprecedented flexibility for the user and, except for the analog front end, is completely digital, fully programmable and is housed in a pressure case capable of bottom (upward looking), mooring or shipboard (downward looking) mounting configurations. The aluminum pressure case is capable of deployments to $200 \mathrm{~m}$ with connectors for telemetry and power if real time data are required. The WCP can ping at up to $2 \mathrm{~Hz}$ with an acoustic range resolution of $3 \mathrm{~cm}$ and is capable of digitizing four samples per acoustic range bin. The maximum probing depth, depending on the water column properties, is $200 \mathrm{~m}$ and the WCP is capable of storing data internally for up to 3 weeks at maximum ping and sampling rates. Data can be transferred realtime via a $10 \mathrm{Mbit} / \mathrm{s}$ 10Base2 Ethernet link to a surface system or local area network for viewing and/or data storage.

The system can also be controlled remotely over the local area network. For self-contained autonomous insitu deployments, internally recorded data can be recovered from the WCP at very high rates to a storage system over a high speed Ethernet connection. The WCP is designed to work with other acoustic instruments like the RDI Sentinel series ADCPs. The high resolution WCP sonar data complements the ADCP's velocity data creating a very powerful analytical acoustic profiling tool. In September 1998, a prototype of the instrument was deployed near Istanbul in the Bosporus (Istanbul Bogazi) strait. The WCP was co-deployed, in roughly $62 \mathrm{~m}$ of water, with an RDI $300 \mathrm{kHz}$ Sentinel Workhorse ADCP to image the two layered stratified flow in the Bosporus.

\section{Introduction}

\section{A. Overview}

In the past acoustical instrumentation for imaging the water column have for the most part consisted of large, heavy instruments requiring large amounts of power and operator attention to make them work. They were manufactured with virtually all analog parts and have used the same basic design methodology for many years. Until recently, data acquisition for these acoustic instruments has been with an analog strip chart recorder. This technology, which has come to be known as the "echo-sounder", has undergone a significant technological transformation over the past 5 years. This has been largely due to advancements in low power digital electronics and display technologies which have been incorporated into modern Water Column Profilers (WCPs). However most commercially available WCPs are still quite large and are still based on the same functionality as the original echo sounders. For this reason most modern WCPs, although they incorporate many of the new technologies, follow the traditional configuration, being ship mounted and configured to work in a downward looking orientation.

\section{B. A New Design Approach}

With the recent explosion in digital, low power, microprocessor, storage, data acquisition and communications technologies it is finally possible to reduce the WCP to a size where it can be deployed remotely in a non-conventional manner. An acoustical $200 \mathrm{kHz}$ high resolution, digital, programmable Water Column Profiler (WCP) has been developed incorporating these new technologies and, except for the analog front end, is completely digital, fully programmable and is housed in a pressure case capable 


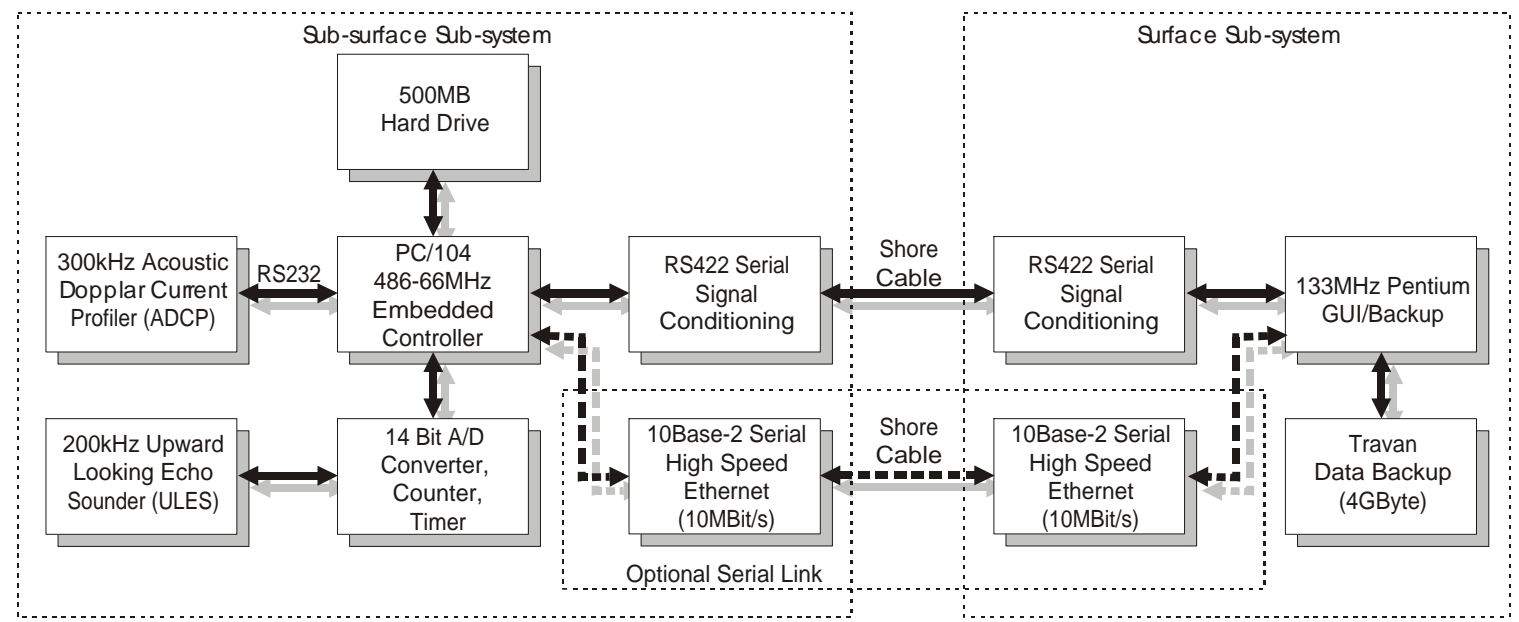

Figure 1: Upward looking water column profiler block diagram

of bottom (upward looking), mooring or shipboard (downward looking) mounting configurations. The aluminum pressure case is capable of deployments to $200 \mathrm{~m}$ with connectors for telemetry and power if real time data is required.

\section{Implementation}

The Upward Looking WCP comprises two sub-systems; a sub-surface section and a surface section (see Figure 1). The sub-surface sub-system resides in a pressure case capable of deployments to $225 \mathrm{~m}$ and can be deployed in either bottom-mounted, moored or surfacemounted configurations for use in either upward or downward modes of operation. The surface sub-system, connected to the sub-surface sub-system via cable, is a station where an operator can control the sub-surface sub-system and view data in real time. It also serves as a mass storage station enabling the overall system to have very long deployment periods. The sub-surface sub-system receives power from either the surface subsystem, for real time deployments or from batteries for autonomous deployments. The surface sub-system can be either ship- or shore- mounted.

The WCP is based on a $200 \mathrm{kHz}$ analog sonar transceiver card capable of pulse repetition (ping) rates of up to $2 \mathrm{~Hz}$ with an acoustic range resolution of $3 \mathrm{~cm}$. The transceiver card compensates for geometric spreading loss with Time Varying Gain (TVG) ranging from 10.3 to $90.3 \mathrm{~dB}$ and has a $3 \mathrm{~dB}$ bandwidth of roughly $6 \mathrm{kHz}$. The card is capable of producing $40 \mathrm{Wrms}$ which yields an effective range of approximately $200 \mathrm{~m}$, depending on the conditions in the water column. The sonar card output is digitized at 14 bit resolution and the data is sent to a PC/104 $66 \mathrm{MHz} 486$ embedded controller. The digitizer is capable of digitizing four samples per acoustic range bin resulting in about 133 samples $/ \mathrm{m}$. This sampling density gives the WCP the ability to produce high resolution images. The data can be stored internally to a 500MB hard drive, if the system is to be deployed in a self-contained autonomous in-situ mode. The drive capacity allows 3 weeks of internal data storage at maximum ping and sampling rates. For an insitu real-time deployment, data from the sub-surface sub-system may be sent via a suitable serial communication link to the surface sub-system for real time display and storage. The serial communication link can be either RS422 or high speed (10 Mbit/sec) 10Base-2 Ethernet through a suitable shore cable. In this configuration the system is controlled remotely over a local area network formed with the serial high speed Ethernet link or through serial commands through the RS422 serial link.

The WCP is designed to work with the $300 \mathrm{kHz}$ RDI Workhorse series Acoustic Doppler Current Profilers (ADCPs). The ADCP interfaces directly to the 486 embedded PC/104 controller through the RS232 serial port (Figure 1). In the in-situ autonomous deployment mode its data is written to its own internal memory, 80 Mbytes total, but can also be sent to the embedded controller to be stored on the local hard drive or sent up to the surface sub-system for display and storage. The ADCP can be programmed either before the deployment, for in-situ autonomous deployments, or can be programmed while the system is deployed during real time deployments. It is possible to communicate directly with the ADCP from the surface sub-system through the Graphic User Interface (GUI). Once the WCP and the ADCP are running the two systems operate independently and, because of the difference in operating frequencies, do not interfere with each other. If the ADCP is programmed to pass data to the embedded controller, the controller takes the ADCP data, along with each ping of WCP data, and puts it in packet form. Along with header and checksum information, the packet is sent up to the surface sub-system via the serial communication link. The surface sub-system then checks for transmission errors, unpacks the data, displays the WCP data and stores both the WCP and ADCP data to hard drive. For self-contained autonomous 
in-situ deployments, internally recorded data can be recovered from the WCP at very high rates to a storage system over a high speed Ethernet connection.

\section{Sample Results}

In September 1998, a prototype of the instrument was deployed near Istanbul in the Bosporus (Istanbul Bogazi) Strait (see Figure 2). The WCP was co-deployed, in 62m of water, with an RDI $300 \mathrm{kHz}$ Sentinel Workhorse ADCP to image the two layered stratified flow in the Bosporus. The Bosporus provides an extremely challenging and interesting site to image. A multi layered flow exists, principally driven by the prevailing north winds, which force the fresh Black Sea water to flow south into the Sea of Maramara. This, in turn, sets up a counter flow where the dense salty Mediterranean Sea water flows north, underneath the less dense fresh Black Sea water, into the Black Sea. This multi layered flow exchange is responsible, in part, for the transport of salt out of the Mediterranean Basin [1].

Figure 3 shows the deployment configuration in the Bosporus. At the deployment site the Bosporus is $825 \mathrm{~m}$ wide and about $65 \mathrm{~m}$ deep with the deepest part of the channel being on the west side. A $700 \mathrm{~m}$ cable was run from the instrument package to the surface sub-system on the east shore, near the Anadolu Hisari (an old fortress). The WCP was configured with a $68.08 \mathrm{~m}$ range, a ping rate of $0.5 \mathrm{~Hz}$ and a digitization rate of 10 $\mathrm{kHz}$.

Figures 4 and 5 show an 8 minute, 55 second section of ADCP and WCP data, respectively. Figure 5 also shows a plot of both sound speed and sigma-t vs. depth. Sigma-t varies from 11.5 in the upper layer (Black Sea water) to 28 in the lower layer's Mediterranean Sea water. There is a significant sound channel in the interface region between the two layers. Figure 4 displays data from the ADCP, illustrating the strong shear in the flow. Current speeds in the upper layer reach nearly $1 \mathrm{~m} / \mathrm{s}$ to the south and in the lower layer

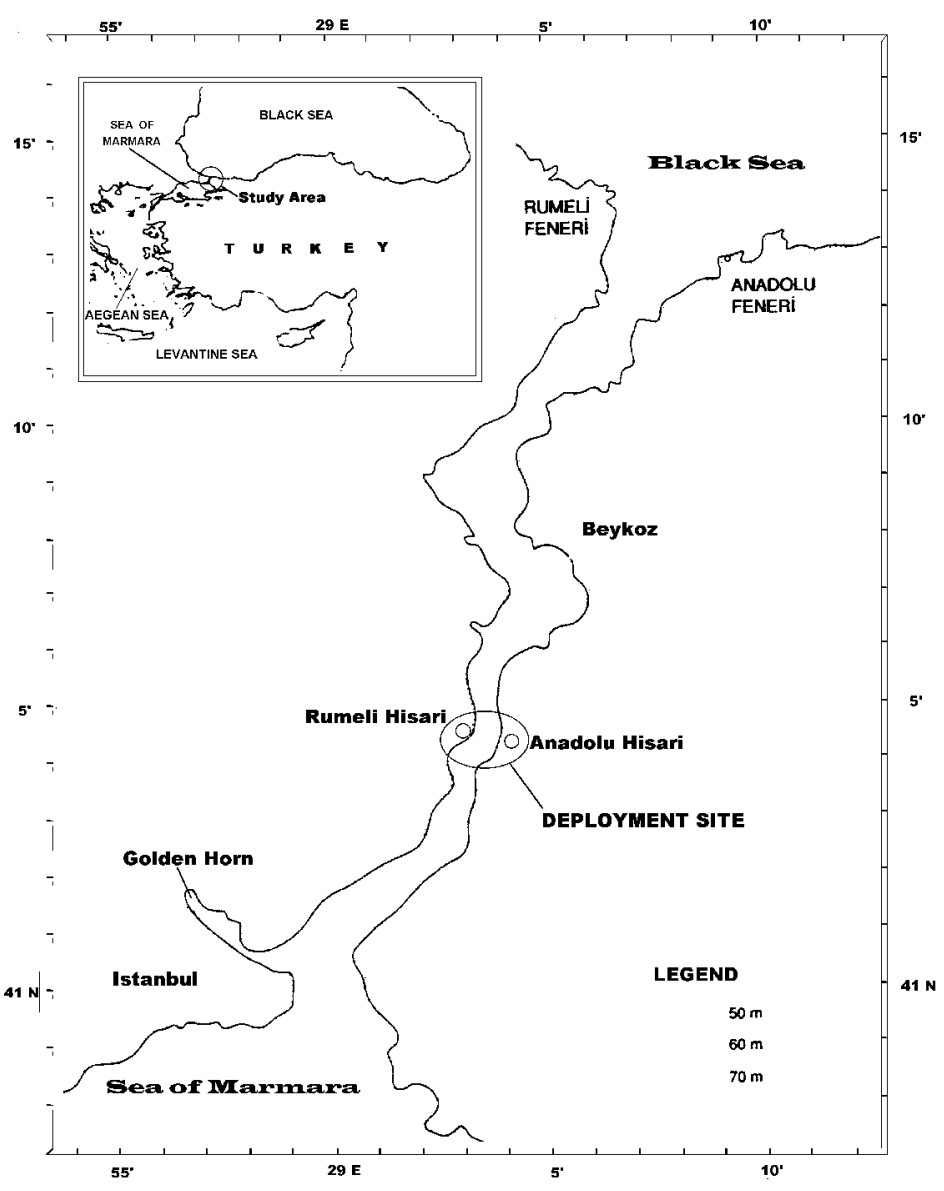

Figure 2: Map showing the deployment site near Istanbul in the Bosporus (Istanbul Bogazi).

exceed $1 \mathrm{~m} / \mathrm{s}$ to the north (bottom right image). The interface region can be discerned in the intensity image on the left of Figure 4 although the detail is poor. In contrast, details of the interface region can clearly be seen in the high resolution WCP image shown in Figure 5. Here details of the fine structure that exists in the interface region are clearly evident. One interesting note is that the last part of the record shows a wave-like structure starting on the interface. This corresponds to a bow wave being impressed on the interface during the

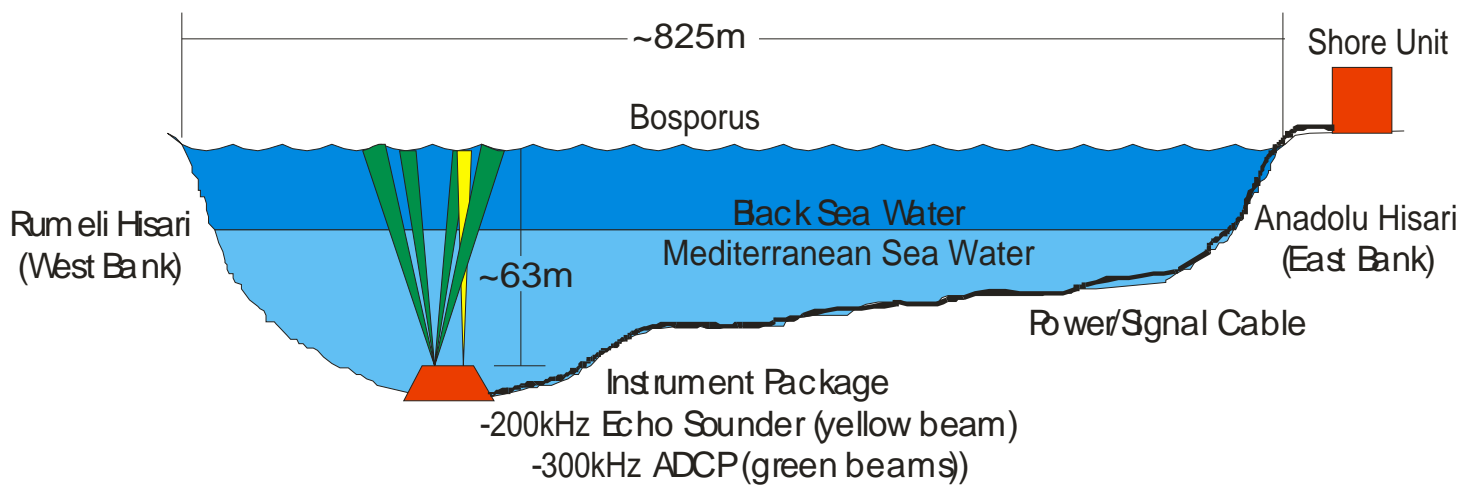

Figure 3: Prototype WCP deployment in the Bosporus. 


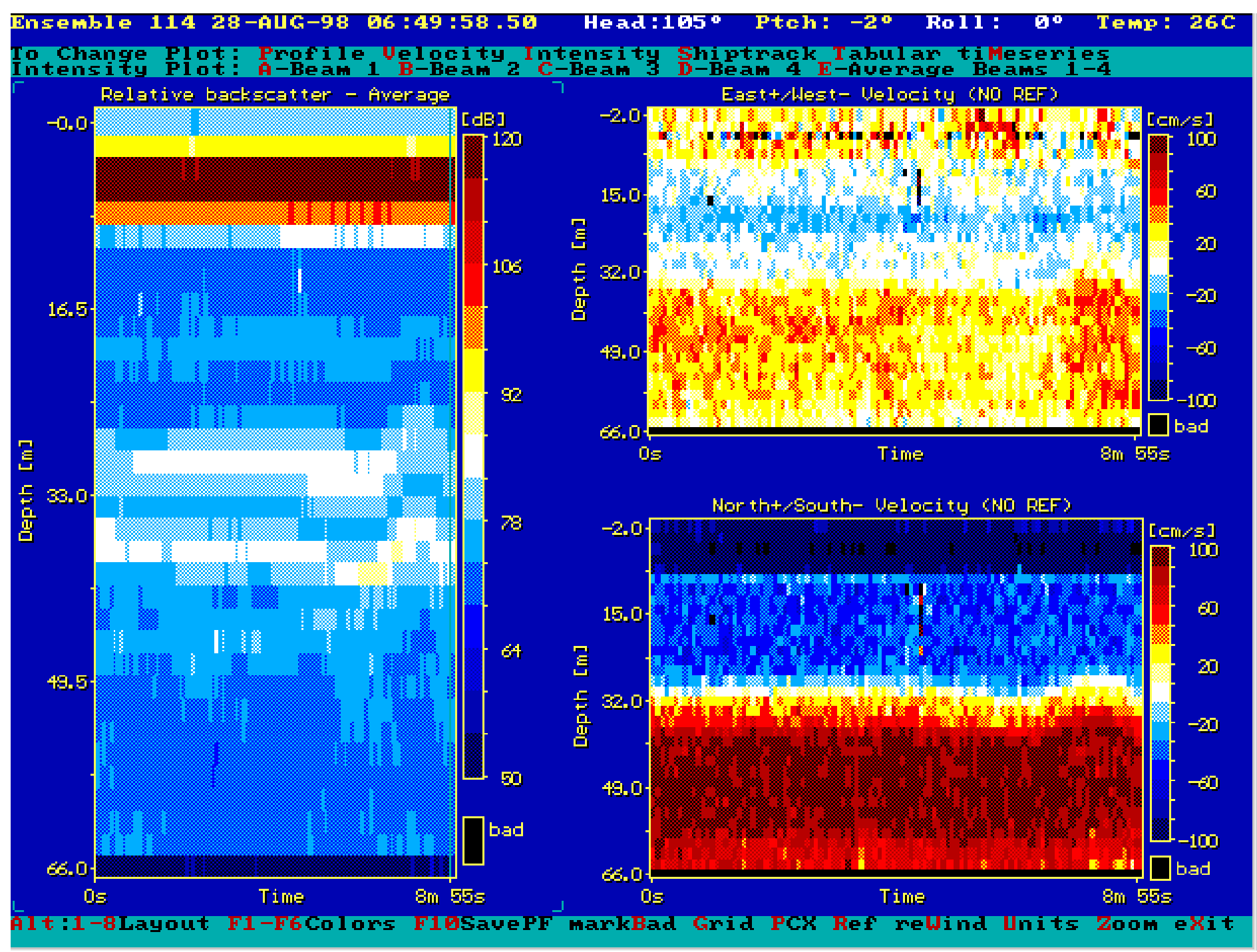

Fig. 2: ADCP data record for 8 minutes, 55 seconds at the Bosporus site.
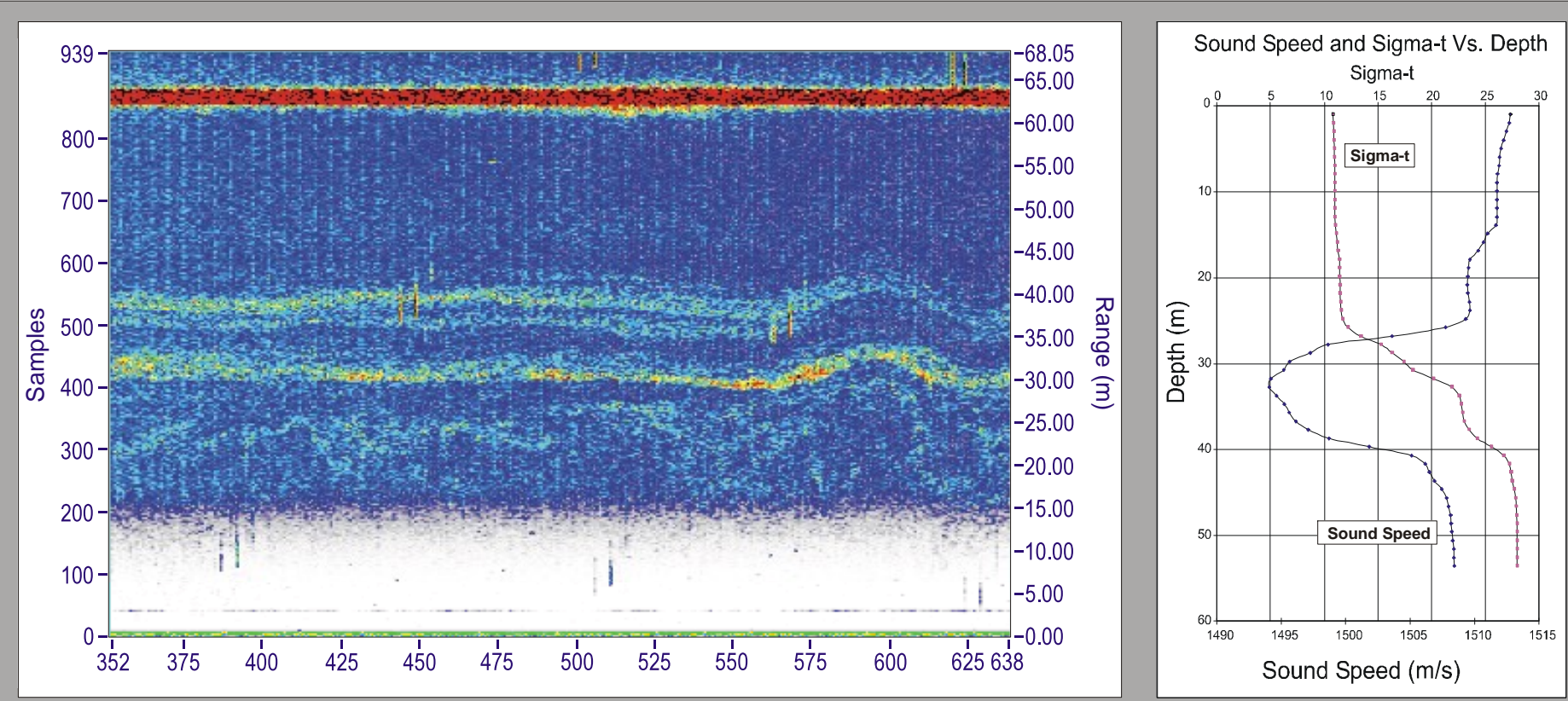

Figure 3: WCP data record for the same 8 minute, 55 second period as the ADCP data in Fig. 4. 
passage of a large tanker. Short sections of vertical lines are seen at intervals in the image which the authors believe originate from the ship's sounder as it was passing overhead. These are not evident in the rest of the record before or after the passage of the tanker.

\section{Conclusions}

With the tremendous burst of technology improvements over the past 5 years it is now possible for a new highly flexible, fully digital, low power, compact Water Column Profiler to be constructed. This high resolution acoustic imaging instrument is capable of operating in new modes and deployment schemes impossible only a few years ago. The high resolution WCP sonar data complements the ADCP's velocity data creating a very powerful analytical acoustic profiling tool.

\section{References}

[1] U. Unluata, T. Oguz, M. A. Latif, E. Ozsoy, "On the physical oceanography of the Turkish straits", L. J. Pratt (ed.), The Physical Oceanography of Sea Straits, pp. 25-60, Kluwer Academic Publishers (Neth), 1990. 